\title{
Functional Photonic Devices based on Silicon Wire Waveguide
}

\author{
Koji Yamada, Hiroshi Fukuda, Toshifumi Watanabe, Tai Tsuchizawa, Tetsufumi Shoji, Sei-ichi Itabashi \\ NTT Microsystem Integration Laboratories \\ 3-1, Morinosato-Wakamiya, Atsugi, 243-0198 Japan
}

\begin{abstract}
We demonstrate our recent progress in functional photonic devices based on a silicon wire waveguide. The propagation loss of the waveguide has been reduced to around 2 $\mathrm{dB} / \mathrm{cm}$, and the inorganic low-loss fiber coupling structure has been completed. For passive functions, we have developed ring resonators and apodized lattice filters for add/drop filtering devices. The ring resonators realized very high $Q$ values of around 20,000 . The lattice filters realized large free spectral range and low cross-talk. For active, or nonlinear function, we demonstrate all-optical wavelength conversion based on the four-wave-mixing effect. The conversion efficiency was $-12 \mathrm{~dB}$, and clear eye-patterns of the converted signal are observed at $\mathbf{1 0}$ Gbps operation.
\end{abstract}

\section{INTRODUCTION}

A silicon wire waveguide is a promising platform for high-density integration of optical circuits [1]. Due to its very high refractive index contrast, or its strong light confinement, the core size of the waveguide for single-mode propagation is a few hundred nanometers and its bending radius is a few micrometers for a $1500-\mathrm{nm}$ infrared system. The waveguide with an ultra-small core and sharp bends allows the construction of ultra-small optical systems, and realizes a large-scale integration of optical circuits. Moreover, the waveguide with an ultra-small core realizes an ultra-high optical power density, as much as 1000 times that in a conventional single-mode fiber. Consequently, nonlinear optical effects will be enhanced so that they can be used as practical photonic devices.

In this work, we demonstrate some of our silicon wire based functional devices. For passive functions, we show add/drop filters suitable for WDM communication systems. For active, or nonlinear functions, we show four-wave-mixing (FWM) based on an all-optical wavelength conversion.

\section{FUNDAMENTAL CHARACTERISTICS}

The cross-sectional structure of the silicon wire waveguide is shown in Figure 1. The core size was $400 \sim 460$ by $200 \mathrm{~nm}$ to maintain the single-mode condition. The propagation loss has been improved by reducing the side-wall roughness to less than $2 \mathrm{~nm}$ [2]. The core size also affects propagation losses. Figure 2 shows the relation between measured propagation losses and core widths. As shown, the propagation loss was reduced to $2 \mathrm{~dB} / \mathrm{cm}$ by increasing the core width to $460 \mathrm{~nm}$.

Problems in coupling to an external fiber have been solved by introducing a spot-size converter (SSC) with an inverse taper [3]. The structure of the converter is shown in Figure 3.

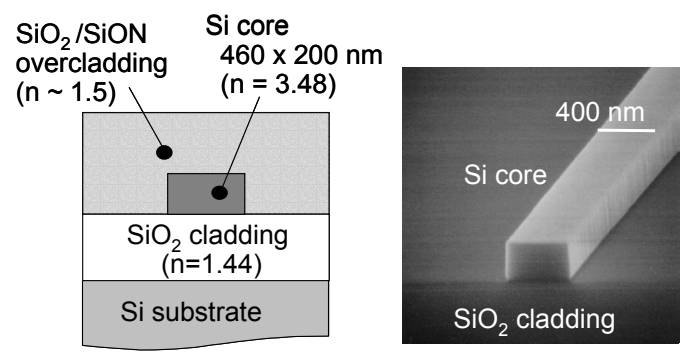

Fig. 1. Structure of silicon wire waveguide.

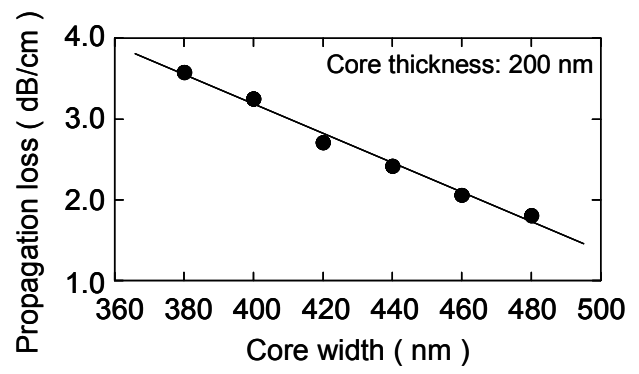

Fig. 2. Relationship between propagation losses and core width.

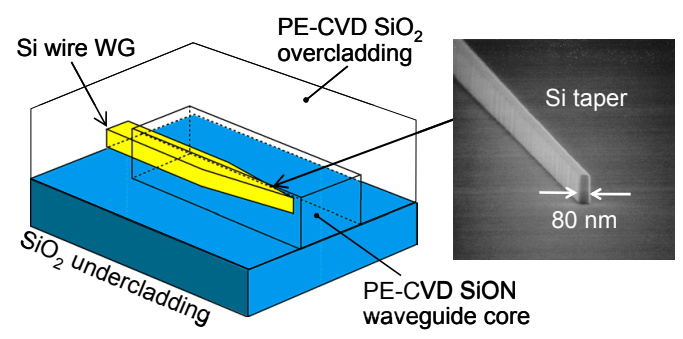

Fig. 3. Structure of spot size converter.

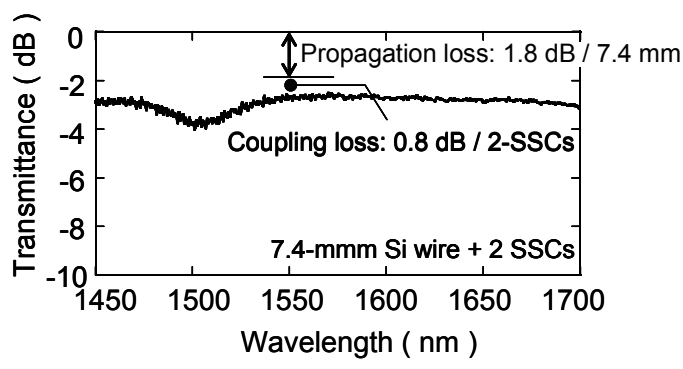

Fig. 4. Transmission spectrum of a silicon wire waveguide with SSCs. 
A $300 \mu \mathrm{m}$-long silicon taper with $80 \mathrm{~nm}$ tip is located in a 3 by $3 \mu \mathrm{m}$ single-mode silica waveguide [2]. Figure 4 shows a transmission spectrum of a $7.4 \mathrm{~mm}$-long silicon wire waveguide with SSCs. Using fibers with a $4.3 \mu \mathrm{m}$ field diameter, the coupling loss was around $0.5 \mathrm{~dB} /$ coupling at $1550 \mathrm{~nm}$ and the applicable bandwidth was over $250 \mathrm{~nm}$. The $\mathrm{SSC}$ is made of inorganic materials; therefore, there is no deterioration even at $20 \mathrm{dBm}$ of input power.

\section{PASSIVE DEVICES}

\section{A. Ring Resonator}

An add/drop filtering device is one of the most appealing passive optical devices. For a narrow-band add/drop device, we have developed high-Q ring resonators. Figure 5 shows a photograph of a ring resonator with a $10 \mu \mathrm{m}$ radius, and Figure 6 shows the measured transmission spectrum. The resonance bandwidth is very narrow as $80 \mathrm{pm}$, and the quality factor $\mathrm{Q}$ was as large as 20000 . In spite of its very high Q value, the ring has a practical transmittance of $-3 \mathrm{~dB}$. Such a high-Q and high-transmission resonator might be used as a channel drop filter for dense WDM systems.

Figure 7 shows power-density enhancement in the ring resonators. The enhancement factors were estimated from the measured $Q$ values and transmittances. As shown, the power density is significantly increased in ring resonators. In other words, a silicon wire based ring resonator can be a promising platform for nonlinear optical devices.
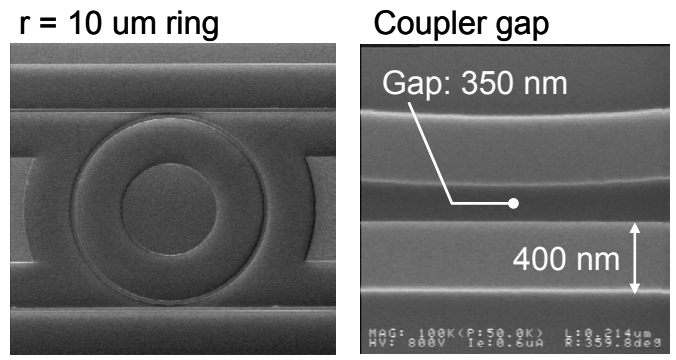

Fig. 5. Structure of a ring resonator filter.

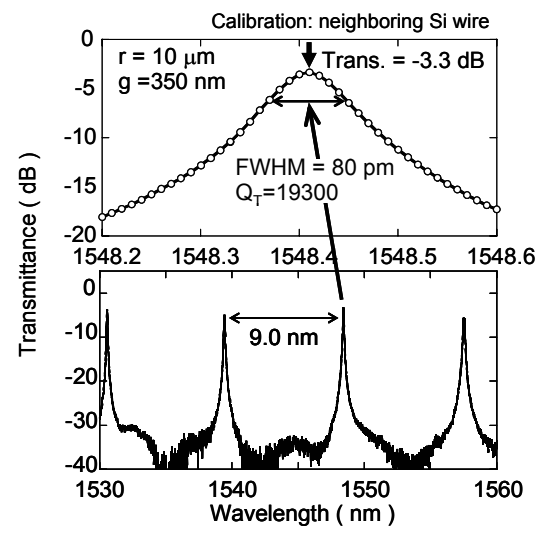

Fig. 6. Drop port spectrum of a ring resonator filter.

\section{B. Apodized Lattice Filter}

As shown in Figure 6, the FSR of the ring resonator is narrow as around $10 \mathrm{~nm}$. For add/drop filters with a large FSR, we have already developed integrated lattice filters $[1,2,4]$. However, the channel cross-talks were around $-10 \mathrm{~dB}$, and should be drastically reduced. To reduce cross-talk, we have developed an apodized lattice structure. Figure 8 shows a part of the apodized lattice structure. A snake-like delay line is made of an arc with a radius of $2.5 \mu \mathrm{m}$, and it encounters a straight section periodically, where directional couplers are constructed. The lengths of the couplers are modified for apodization.

Figure 9 shows the measured transmission spectrum of the drop ports for apodized and non-apodized filters. These filters exhibit beautiful channel dropping characteristics. The extinction ratio to sidelobe peaks, that determines the cross-talk, was greatly improved by the apodization. The apodization technique is very important in realizing low cross-talk filters for practical WDM systems.

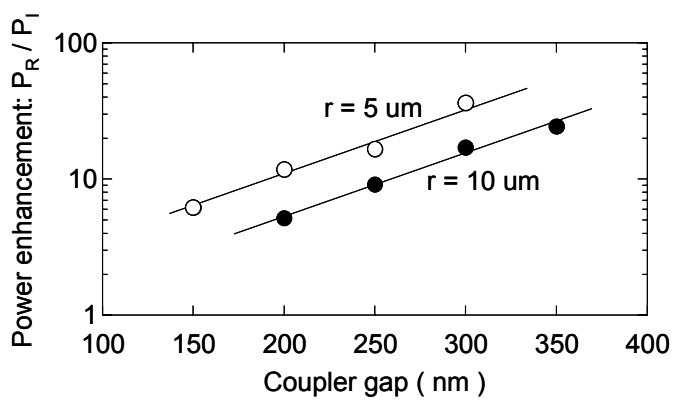

Fig. 7. Power density enhancement in various ring resonators. $\mathrm{P}_{\mathrm{R}}$ : Power density in the ring, $\mathrm{P}_{\mathrm{I}}$ : Power density in the input bus-line

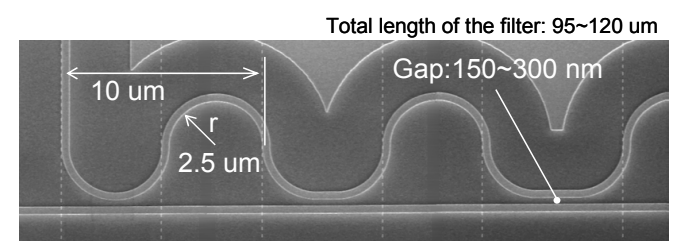

Fig. 8. Structure of a typical apodized lattice filter.

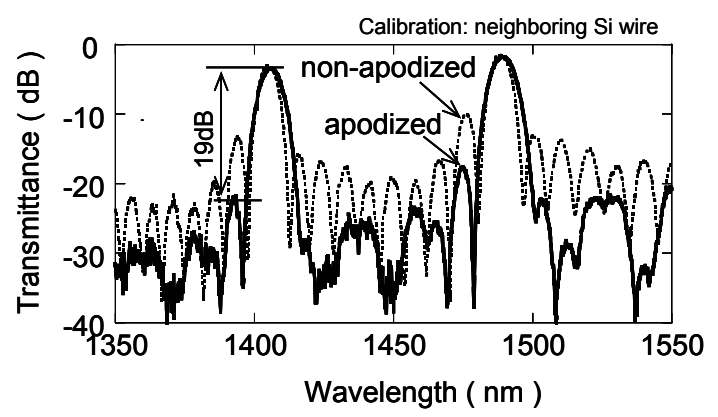

Fig. 9. Drop port spectra of lattice filters. 


\section{NONLINEAR DEVICES}

We have observed various useful nonlinear functions, such as four-wave-mixing (FWM), two-photon-absorption (TPA), etc., enhanced in silicon wire waveguides[2]. We demonstrate here FWM-based all-optical wavelength conversion, which might be an important technique in all-optical routing system.

The experimental setup is shown in Figure 10. The signal light was modulated to a $10 \mathrm{Gbps} \mathrm{NRZ}$ data stream by a lithium-niobate modulator. The pump was in continuous-wave (CW) operation. The detuning between signal and pump light is $0.22 \mathrm{THz}$. The peak power of the signal input was about $5 \mathrm{~mW}$. Pump input was $100 \mathrm{~mW}$ CW. Both lights were adjusted to TE polarization, and injected into a $2.9 \mathrm{~cm}$-long silicon wire waveguide with a 460 by $200 \mathrm{~nm}$ core. The propagation loss of the waveguide is $2.4 \mathrm{~dB} / \mathrm{cm}$. The coupling loss is about $0.5 \mathrm{~dB}$; therefore, the power density in the waveguide is over $100 \mathrm{MW} / \mathrm{cm}^{2}$.

Figure 11 shows the output spectrum at the exit of the waveguide. We can obviously see a peak of converted light in the spectrum. The fine structures in the peak are sidelobes for 10Gbps modulation. Conversion efficiency defined as the peak level ratio between the signal and converted lights was about $-12 \mathrm{~dB}$. This conversion efficiency is large enough for practical data processing. As shown in Figure 12, we have easily obtained clear eye patterns of a 10Gbps PRBS data stream for converted light. Presently the conversion efficiency is mainly limited by the propagation loss of the waveguide. Theoretical estimation shows that the conversion efficiency reaches around $-6 \mathrm{~dB}$ for $1 \mathrm{THz}$ detuning using a waveguide with $1 \mathrm{~dB} / \mathrm{cm}$ propagation loss [5].

\section{SUMMARY}

Using a silicon wire waveguide with low propagation loss and efficient fiber coupling, we have developed various functional photonic devices. The superior characteristics of the ring resonators and success in the sophisticated apodization of the lattice filters mean that silicon wire based passive devices can be applied in practical use soon. The practical conversion efficiency obtained in the FWM based wavelength conversion means that the high-power-density effect in a silicon wire waveguide can be a breakthrough to silicon-based active devices.

\section{REFERENCES}

[1] K. Yamada, T. Tsuchizawa, T. Watanabe, J. Takahashi, E. Tamechika, M. Takahashi, S. Uchiyama, T. Shoji, H. Fukuda, S. Itabashi, H. Morita, IEICE Trans. Electron., vol. E87-C, pp.351-358, 2004.

[2] T. Tsuchizawa, K. Yamada, H. Fukuda, T. Watanabe, J. Takahashi, M. Takahashi, T. Shoji, E. Tamechika, S. Itabashi, H. Morita, IEEE Jounal of Selected Topics Quantum Electronics, vol. 11, pp.232-240, 2005.

[3] T. Shoji, T. Tsuchizawa, T. Watanabe, K. Yamada, H. Morita, Electron Lett. vol. 38, pp.1669-1670, 2002.

[4] K. Yamada, T. Shoji, T. Tsuchizawa, T. Watanabe, J. Takahashi, S. Itabashi, Opt. Lett., vol. 28, pp.1663-1664, 2003.

[5] H. Fukuda, K. Yamada, T. Shoji, M. Takahashi, T. Tsuchizawa, T. Watanabe, J. Takahashi, S. Itabashi, to be published in Optics Express.

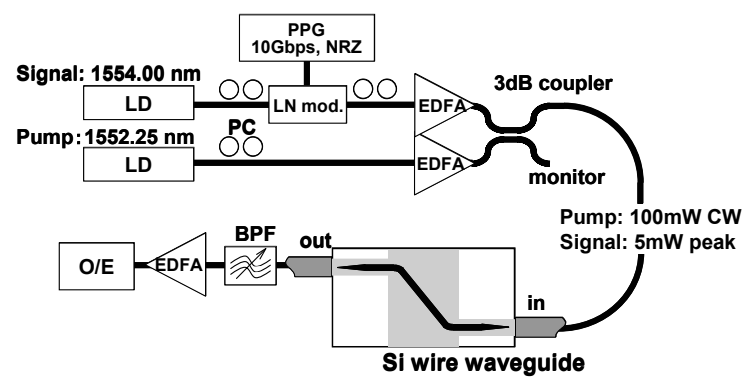

Fig. 10. Experimental setup for FWM based wavelength conversion.

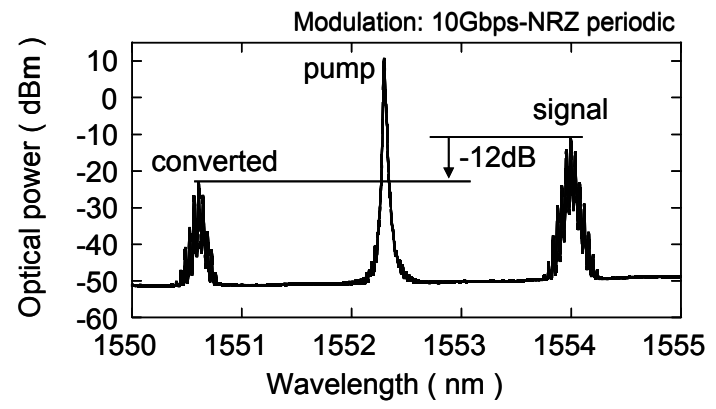

Fig. 11. Output spectrum of wavelength conversion experiment.

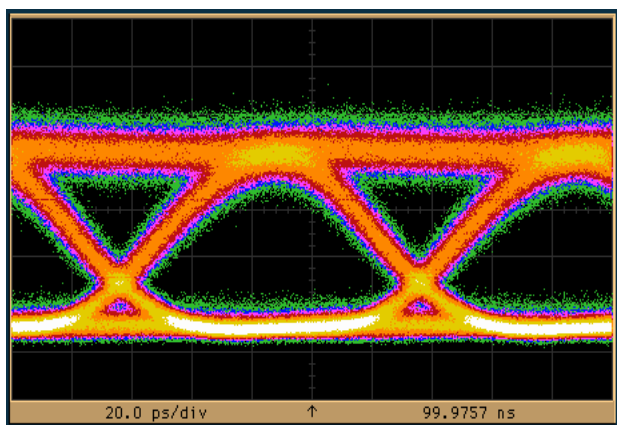

Fig. 12. Eye pattern of converted signals. 10Gbps-NRZ PRBS, mark ratio: $1 / 2$. 